\title{
ANÁLISE DAS PROPRIEDADES MECÂNICAS DO AÇO BH
} 180*

\author{
Géssica Seara Faria ${ }^{1}$ \\ Luxmar Alves Xavier ${ }^{2}$ \\ Ricardo Evangelista César ${ }^{3}$ \\ Rudd Gullit Francisco dos Santos ${ }^{1}$ \\ Victor Hugo Andrade da Silva ${ }^{1}$
}

\section{Resumo}

A corrida pela alta produtividade, a demanda pela otimização de processos, que proporciona maior economia de combustível, impulsiona o desenvolvimento de estruturas veiculares de menor peso. $\mathrm{O}$ aço Bake Hardening $(\mathrm{BH})$ é um aço de ultra baixo carbono estabilizado ao titânio, que apresenta átomos de carbono em solução sólida ocupando posições intersticiais. Este passa por um tratamento realizado durante o trabalho de pintura do veículo, permitindo que as chapas sejam endurecidas pelo efeito $\mathrm{BH}$, conferindo resistência à endentação. Neste estudo um aço $\mathrm{BH}$ foi analisado em quatro condições de processo: laminação a quente, laminação a frio, recozimento e encruamento, com o objetivo de estudar os efeitos da variação destes parâmetros nas propriedades mecânicas do material, através dos ensaios de tração, obtendo resultados como valor de alongamento adquirido e dureza.

Palavras-chave: Bake hardening; Estruturas veiculares; Propriedades Mecânicas.

\begin{abstract}
ANALYSIS OF MECHANICAL PROPERTIES OF STEEL BH 180
Abstract

The race for high productivity, the demand for process optimization, providing improved fuel economy, drives the development of vehicle structures of lower weight. The Bake hardening steel $(\mathrm{BH})$ and an ultra low carbon steel stabilized titanium, which has carbon atoms in solid solution occupying interstitial positions. This undergoes a treatment carried out during the vehicle paint job, allowing the plates are hardened by $\mathrm{BH}$ effect conferring resistance to indentation. In this study, a steel $\mathrm{BH}$ was analyzed in four process conditions: hot rolling, cold rolling, annealing and hardening, in order to study the effects of varying these parameters on the mechanical properties of the material, through tensile tests, obtaining results for granted elongation value and hardness.
\end{abstract}

Keywords: Bake hardening; Vehicle structures; Mechanical Properties.

1 Graduanda em Engenharia Metalúrgica, Centro Universitário do Leste de Minas Gerais - Unileste, Coronel Fabriciano, Minas Gerais, Brasil.

2 Bacharel em Engenharia Metalúrgica, Supervisor, Laboratório de Testes Mecânicos, Usiminas S/A Ipatinga, Minas Gerais, Brasil.

3 Graduando em Engenharia Metalúrgica, Supervisor, Laminação a Frio, Usiminas S/A Ipatinga, Minas Gerais, Brasil. 


\section{INTRODUÇÃO}

\subsection{Definições dos Aços IF}

A sigla "IF", do inglês "interstitial-free", expressa a ausência de elementos intersticiais na matriz ferrítica de alguns aços, podendo definir aços IF como aços de composição com ultra baixo carbono e nitrogênio, os quais são retirados do estado de solução sólida podendo formar precipitados pela adição de elementos formadores de carbeto e nitreto tais como o titânio e o nióbio, deixando assim, em juz ao nome, 0 aço livre de elementos intersticiais [1,2].

Não existe uma classificação padronizada de aços IF. Geralmente, eles são distinguidos pelo elemento que estabiliza os solutos. Por exemplo, se fala em aços IF estabilizados por titânio, por nióbio ou por ambos. Podem existir ainda aços IF de alta resistência e aços IF "envelhecíveis" ou "Bake Hardening" durante a cura da pintura a quente de peças conformadas. Quando o endurecimento por solução sólida é promovido por adições de fósforo, este aço é normalmente chamado de IF refosforado (IF-P) [2].

Para cada tipo de aço IF a precipitação será diferente e dependente das diferenças na composição química e no processamento termomecânico, o que pode afetar a microestrutura e textura final do material [2].

\subsection{Características dos Aços $\mathrm{BH}$}

Os aços bake hardening $(\mathrm{BH})$ consistem principalmente de ferrita contendo um mínimo de carbono em solução sólida. Eles são usados no processo automotivo principalmente na conformação de painéis externos e posteriormente são pintados na faixa de temperatura de $150^{\circ} \mathrm{C}$ a $200^{\circ} \mathrm{C}$, o carbono é dissolvido e difunde para alguns pontos livres na migração das discordâncias na operação de conformação [3]. Apresentam uma conformabilidade muito boa antes do tratamento térmico e podem suportar estampagens moderadas e profundas e, após tratamento térmico, podem alcançar níveis de resistência mais altos. Sendo assim, possuem baixas propriedades mecânicas, na condição de recém-produzidos, adequadas aos processos de estampagem e conformação, que após a pintura, exibem aumento considerável dessas propriedades, tornando a peça de espessura fina adequada ao uso e melhor resistência a endentação [3].

Para que um aço apresente efeito consistente de bake hardening é necessário que ele tenha teor de $\mathrm{C}$ solúvel de, no mínimo, 0,0005\% (menor que $25 \mathrm{ppm}$ ), para que o aumento de resistência mecânica após o envelhecimento seja igual ou superior a 30 $\mathrm{MPa}$. Por outro lado, teores excessivos de $\mathrm{C}$ solúvel são inconvenientes: acima de $0,0015 \%$ o efeito de bake hardening torna-se mais intenso, ultrapassando $60 \mathrm{MPa}$, mas também aumenta o risco de ocorrer envelhecimento excessivo do material sob temperatura ambiente antes do prazo especificado para a sua utilização. Isso promove a ocorrência das chamadas linhas de distensão quando da estampagem da chapa, o que pode vir a degradar ou mesmo inutilizar a peça [4].

Chapas finas de aço com efeito de bake hardening e valores de $r$ inferiores a 2,0 podem ser produzidas de duas formas. Na primeira delas um aço com baixo $C(0,02$ a $0,06 \%$ ), acalmado ao Al, é processado numa linha de recozimento contínuo. Esse material é submetido a altas temperaturas de bobinamento no laminador de tiras a quente, o que coalesce os carbonetos e estabiliza o $\mathrm{N}$ na forma de AIN [4]. 
A laminação a frio e o recozimento contínuo resultam na formação de uma intensa textura cristalográfica [111], resultando em produtos com valores de coeficiente de anisotropia $r$ da ordem de 1,5 a 1,7. Parte de seus carbonetos se dissolve durante as fases de aquecimento e encharque no recozimento, obtendo-se assim altos teores de $\mathrm{C}$ em solução. A etapa de super envelhecimento durante o resfriamento tende a estabilizar parte do $\mathrm{C}$ dissolvido na forma de $\mathrm{Fe}_{3} \mathrm{C}$. Logo, é possível controlar as condições dessa última fase do processo de forma que o teor de $\mathrm{C}$ em solução torne- se igual a aproximadamente $0,0010 \%$, proporcionando, assim, níveis adequados de bake hardening [4].

O efeito $\mathrm{BH}$ é uma difusão controlada que resulta da migração de átomos de carbono e nitrogênio em solução na rede para as discordâncias, formando a Atmosfera Cottrel. A formação destas atmosferas faz com que a tensão necessária para causar novamente a deformação plástica aumente, resultando em ganhos no limite de escoamento que podem variar entre 30 e $40 \mathrm{MPa}$ [5].

Com isso, será necessária a aplicação de uma tensão maior para fazer que a discordância se livre da atmosfera ou se mova junto com os átomos. A difusão desses átomos é função do tempo, da temperatura e da quantidade de átomos em solução presente no material. O tamanho de grão e densidade de discordâncias também pode influenciar nesse processo [5].

Entre os aços mais empregados pela indústria automotiva, o $\mathrm{BH}$ se situa em uma faixa aproximada de limite de resistência de 200 a $350 \mathrm{MPa}$ (antes de estampagem e pintura) e alongamento de 30 a 40\%, situando-o em uma faixa de aços de alta ductilidade, mas de baixa a média resistência [6].

Estas características fazem com seja utilizado em peças estampadas que não atuem como reforços estruturais, mas que requeiram resistência à endentação e capacidade de deformar-se amplamente sem sofrer ruptura, tais como portas e paralamas [6].

Conhecido também como "envelhecimento controlado", o efeito bake hardening é um processo estático realizado por tratamento térmico, com o objetivo de aumentar o limite de escoamento do material. Este envelhecimento também ocorre à temperatura ambiente, porém com menor velocidade [6].

\section{MATERIAIS E MÉTODOS}

Para avaliar o comportamento do material ao longo das etapas de laminação, foram realizados ensaios mecânicos e metalográficos nas direções longitudinal e transversal do material. Os corpos de prova (CPs) são provenientes do aço BH 180 nas seguintes etapas de processamento da conformação mecânica, sendo corpos de prova de laminação a quente, laminação a frio, recozimento e laminação de encruamento.

A preparação dos corpos de prova para todos os ensaios de tração seguiu a norma DIN 53504 S2, utilizando oito CPS com as seguintes medidas: a espessura entre $0,99 \mathrm{~mm}$ a $0,84 \mathrm{~mm}$, o comprimento de $80 \mathrm{~mm}$ e a largura de $20 \mathrm{~mm}$. Os corpos de prova foram solicitados para o laboratório de Mecânica e Metalúrgia da Unileste e usinados no próprio laboratório, para garantia da qualidade de bordas, eliminando a região mecanicamente afetada pelo corte, em tesoura guilhotina.

Os ensaios de tração foram realizados em máquina de tração eletromecânica da marca INSTRON, com vídeo-extensômetro. O limite de escoamento foi medido a $0,2 \%$ de deformação, com taxa de deformação de $10 \mathrm{~mm} / \mathrm{min}$ no campo elástico e $30 \mathrm{~mm} / \mathrm{min}$ no campo plástico. O coeficiente de anisotropia (valor $\mathrm{r}$ ) foi obtido entre 
10 a $16 \%$ de deformação permanente, e o coeficiente de encruamento (valor $n$ ) foi medido em $18 \%$ de alongamento.

Os valores de anisotropia e expoente de encruamento foram obtidos pelo modelo matemático da curva tensão x deformação real, conforme a norma NBR 8164, a partir de três direções em relação a direção de laminação, sendo elas a transversal $\left(90^{\circ}\right)$, longitudinal $\left(0^{\circ}\right)$ e diagonal $\left(45^{\circ}\right)$. O resultado final foi dado através da média dos resultados nas três direções avaliadas.

\section{RESULTADOS E DISCUSSÃO}

As seguintes propriedades mecânicas (LE, LR, alongamento, $r$ e $n$, dureza e valor $\mathrm{BH})$ foram medidas nos processos seguintes de laminação a quente, laminação a frio, recozimento e encruamento, revelaram uma dispersão significativa entre os resultados, após análise de variância.

Entre os processos de laminação a quente, como o material não é submetido a grandes esforços mecânicos (figura 1) e não há incremento significativo de temperatura, não há praticamente variação das propriedades mecânicas, o que é confirmado pelos testes realizados nos corpos de prova I0162928. O valor BH (figura 2) apresentou números próximos com ganho efetivo de resistência após deformação e envelhecimento, o valor $\mathrm{n}$ mostra a capacidade que o material possui de ser submetido à deformação e $r$ o comportamento das propriedades ao longo das direções (figura 3).
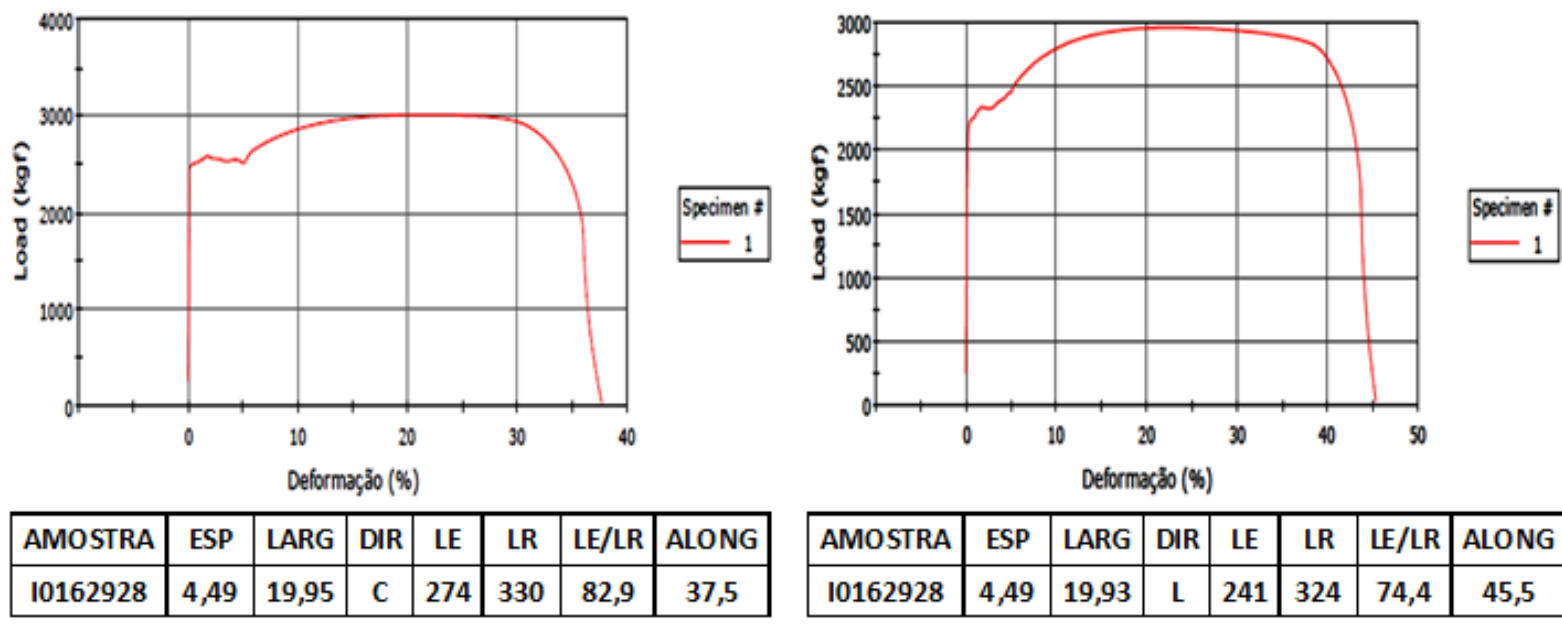

\begin{tabular}{|c|c|c|c|c|c|c|c|}
\hline AMOSTRA & ESP & LARG & DIR & LE & LR & LE/LR & ALONG \\
\hline 10162928 & 4,49 & 19,93 & L & 241 & 324 & 74,4 & 45,5 \\
\hline
\end{tabular}

Figura 1. Curva de tensão deformação de engenharia do aço BH 180 laminado a quente, sentido transversal $(\mathrm{C})$ e longitudinal $(\mathrm{L})$. 


\begin{tabular}{|c|c|c|c|c|c|c|c|c|c|c|c|c|c|}
\hline \multicolumn{14}{|c|}{ Tração BH } \\
\hline \multirow{2}{*}{ Amostra } & \multicolumn{6}{|c|}{ Primeiro estágio } & \multicolumn{6}{|c|}{ Segundo estágio } & \multirow{3}{*}{\begin{tabular}{|c}
$\mathrm{BH}(\mathrm{MPa})$ \\
40
\end{tabular}} \\
\hline & \multicolumn{2}{|c|}{ ESP $1(\mathrm{~mm})$} & \multicolumn{3}{|c|}{ LARG $1(\mathrm{~mm})$} & LE 1 (MPa) & \multicolumn{3}{|c|}{ ESP $2(\mathrm{~mm})$} & \multicolumn{2}{|c|}{ LARG $2(\mathrm{~mm})$} & LE 2 (MPa) & \\
\hline \multirow[t]{2}{*}{10162928} & \multicolumn{2}{|r|}{4,51} & \multicolumn{3}{|c|}{19,92} & 267 & 4,4 & 47 & 19,8 & & & 307 & \\
\hline & \multicolumn{13}{|c|}{ Lankford (RN) } \\
\hline \multirow{2}{*}{ Amostra } & \multirow{2}{*}{ ESP } & \multirow{2}{*}{ LARG } & \multicolumn{3}{|c|}{ Transversal $\left(90^{\circ}\right)$} & \multicolumn{3}{|c|}{ Longitudinal $\left(0^{\circ}\right)$} & \multicolumn{3}{|c|}{ Diagonal $\left(45^{\circ}\right)$} & \multirow{2}{*}{ R Normal } & \multirow{2}{*}{ Valor de $\mathrm{N}$} \\
\hline & & & AL UNIF & $\mathbf{R}$ & $\mathrm{N}$ & AL UNIF & $\mathbf{R}$ & $\mathrm{N}$ & AL UNIF & $\mathbf{R}$ & $\mathrm{N}$ & & \\
\hline 10162928 & 4,5 & 19,9 & 20,2 & 0,7 & 2,1 & 20,28 & 0,6 & 2,2 & 20,39 & 1,1 & 2 & 0,89 & 2,18 \\
\hline \multicolumn{14}{|c|}{ Dureza (HRB) } \\
\hline \multicolumn{3}{|c|}{ Amostra } & \multicolumn{3}{|c|}{ Medição 1} & \multicolumn{3}{|c|}{ Medição 2} & \multicolumn{3}{|c|}{ Medição 3} & \multicolumn{2}{|r|}{ Média } \\
\hline \multicolumn{3}{|c|}{10162928} & \multicolumn{3}{|c|}{58} & \multicolumn{3}{|c|}{60} & \multicolumn{3}{|c|}{59} & \multicolumn{2}{|r|}{59} \\
\hline
\end{tabular}

Figura 2. Resultados da tração $\mathrm{BH}$ após a laminação a quente, resultados de anisotropia (R) e expoente de encruamento $(n)$ e resultados de dureza na escala Rockwell B.
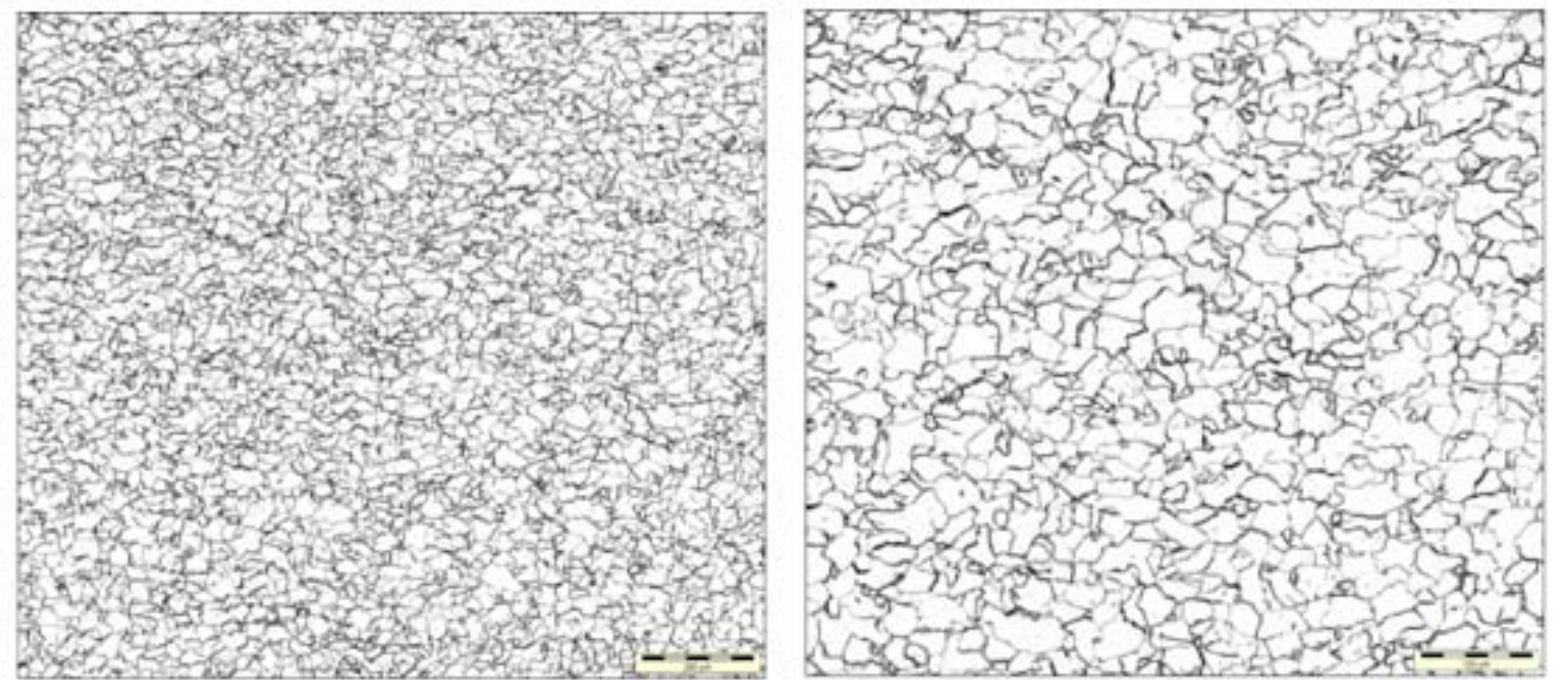

Figura 3. Fotos das microestruturas a partir das amostras laminadas a quente. Ampliação de $100 \mathrm{e}$ 500 Vezes.

Após o processo de laminação a frio há uma grande variação nas propriedades mecânicas. A dureza se eleva consideravelmente e o limite de escoamento (LE) praticamente triplica, se aproximando do limite de resistência, o que indica que o material praticamente não possui zona plástica, devido ao intenso encruamento que foi submetido (figura 4). Na análise metalográfica (figura 6) é possível evidenciar o congestionamento das discordâncias sob a matriz, o que impede novas deformações, explicando o baixo alongamento $(2 \%)$ e a elevada relação elástica (98\%) obtidos no CP I0162930. O valor BH caracterizou-se nulo, pois o material não suportava mais nenhum tipo de esforço mecânico, o que pode ser caracterizado pelo valor zero do coeficiente de encruamento $(n)$ que fornece a capacidade que $o$ material tem de suportar o encruamento e o valor $r$ que apresenta as características das propriedades ao longo das direções (figura 5). 

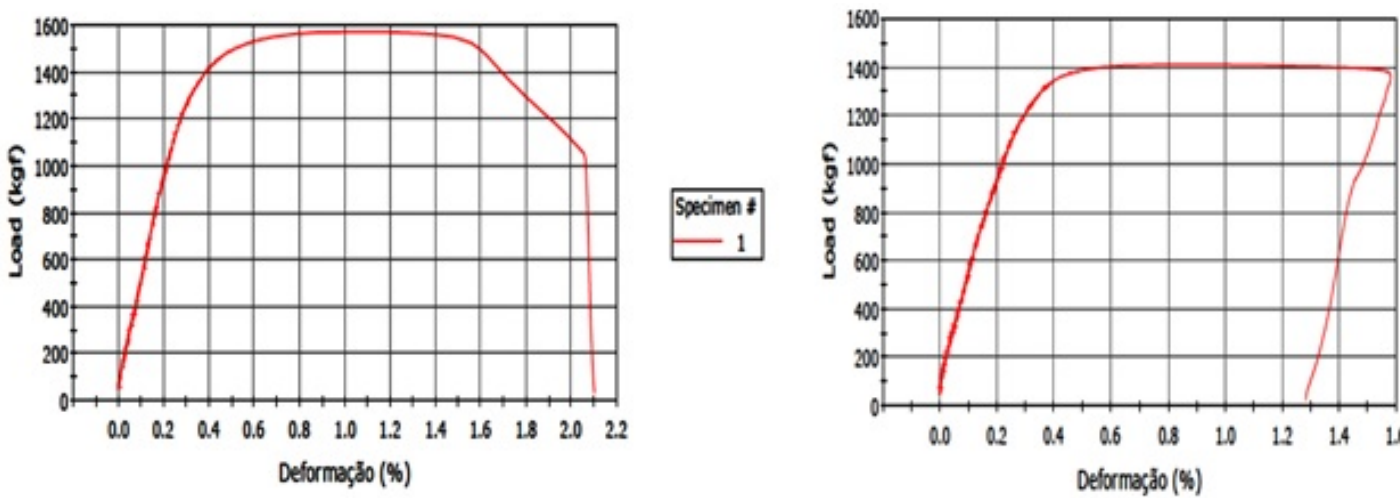

\begin{tabular}{|c|c|c|c|c|c|c|c|}
\hline AMOSTRA & ESP & LARG & DIR & LF & LR & IE/LR & ALONG \\
\hline 10162930 & 0,97 & 19,96 & C & 763 & 795 & 96,1 & 2 \\
\hline
\end{tabular}

\begin{tabular}{|c|c|c|c|c|cc|c|}
\hline AMOSTRA & ESP & LARG & DR & LF & LR & L/LR & ALONG \\
\hline I0162930 & 0,97 & 19,98 & L & 703 & 714 & 98,7 & 1,67 \\
\hline
\end{tabular}

Figura 4. Curva de tensão deformação de engenharia do aço BH 180 laminado a frio, sentido transversal $(C)$ e longitudinal $(L)$.

\begin{tabular}{|c|c|c|c|c|c|c|c|c|c|c|c|c|c|}
\hline \multicolumn{14}{|c|}{ Tração BH } \\
\hline \multirow{2}{*}{ Amostra } & \multicolumn{6}{|c|}{ Primeiro estágio } & \multicolumn{6}{|c|}{ Segundo estágio } & \multirow{2}{*}{$\mathrm{BH}$ (MPa) } \\
\hline & \multicolumn{2}{|c|}{ ESP $1(\mathrm{~mm})$} & \multicolumn{3}{|c|}{ LARG $1(\mathrm{~mm})$} & LF 1 (MPa) & \multicolumn{3}{|c|}{ ESP $2(\mathrm{~mm})$} & \multicolumn{2}{|c|}{ LARG $2(\mathrm{~mm})$} & LE 2 (MPa) & \\
\hline \multirow[t]{2}{*}{10162930} & & 0,97 & \multicolumn{3}{|c|}{19,95} & 723 & \multicolumn{3}{|c|}{0,96} & \multicolumn{2}{|c|}{19,92} & 720 & 0 \\
\hline & \multicolumn{13}{|c|}{ Lankford (RN) } \\
\hline \multirow{2}{*}{ Amostra } & \multirow{2}{*}{ ESP } & \multirow{2}{*}{ LARG } & \multicolumn{3}{|c|}{ Transversal $\left(90^{\circ}\right)$} & \multicolumn{3}{|c|}{ Longitudinal $\left(0^{\circ}\right)$} & \multicolumn{3}{|c|}{ Diagonal $\left(45^{\circ}\right)$} & \multirow{2}{*}{ R Normal } & \multirow{2}{*}{ Valor de $\mathrm{N}$} \\
\hline & & & AL UNIF & $\mathbf{R}$ & $\mathbf{N}$ & AL UNIF & $\mathbf{R}$ & $\mathbf{N}$ & AL UNIF & $\mathbf{R}$ & $\mathrm{N}$ & & \\
\hline 10162930 & 1 & 20 & 0,95 & - & - & 0,74 & - & - & 0,99 & - & - & - & - \\
\hline \multicolumn{14}{|c|}{ Dureza (HRB) } \\
\hline \multicolumn{3}{|c|}{ Amostra } & \multicolumn{3}{|c|}{ Medição 1} & \multicolumn{3}{|c|}{ Medição 2} & \multicolumn{3}{|c|}{ Medição 3} & \multicolumn{2}{|c|}{ Média } \\
\hline \multicolumn{3}{|c|}{10162930} & \multicolumn{3}{|c|}{96} & \multicolumn{3}{|c|}{96} & \multicolumn{3}{|c|}{95} & \multicolumn{2}{|r|}{95,7} \\
\hline
\end{tabular}

Figura 5. Resultados da tração BH após laminação a frio, resultados de anisotropia $(R)$ e expoente de encruamento $(n)$ e resultados de dureza na escala Rockwell B.
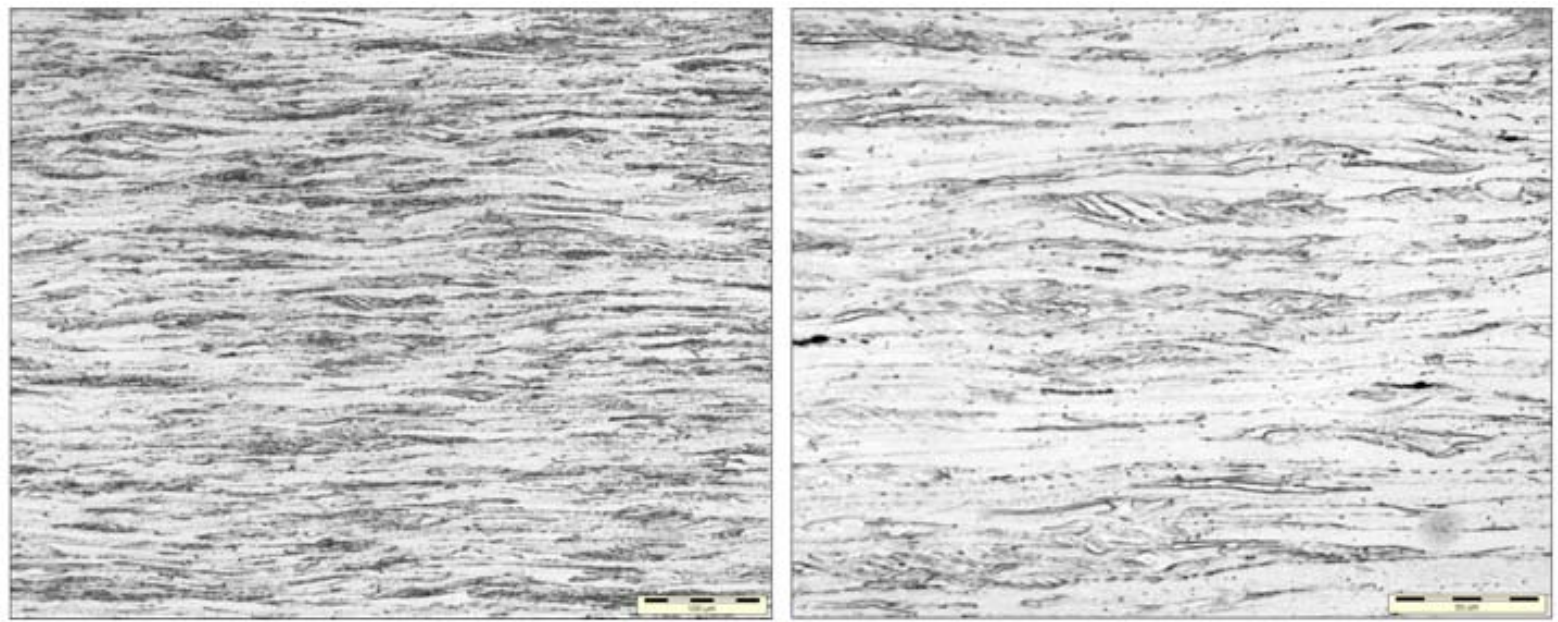

Figura 6. Fotos das microestruturas a partir das amostras laminadas a frio. Ampliação de 500 e 1000 vezes.

Após o ciclo de recozimento total pode-se observar claramente o retorno das propriedades mecânicas para os valores pós-laminação a quente (figura 7). Isso foi possível, pois o processo recristalizou o aço formando novos grãos, maiores e 
ligeiramente equiaxiais, mostrado pelos testes mecânicos e metalográficos realizados do CP 10163845 (figura 9). A dureza e o limite de resistência apresentou valor ligeiramente menor, o que é característico de aços submetidos ao tratamento térmico de recozimento. $\mathrm{O}$ valor de $\mathrm{BH}$ caracterizou-se nulo, pois a diferença entre o limite de escoamento antes e após o tratamento de envelhecimento (uma das etapas do teste $\mathrm{BH}$ ) foi zero, isso devido a morfologia dos grãos favorecer a presença do patamar definido de escoamento que propicia o surgimento de linhas de Luder's (estrias). O valor de $\mathrm{r}$ e $\mathrm{n}$ se eleva consideravelmente, visto que devido a morfologia equiaxial dos grãos o material pode ser submetido uma grande deformação (coeficiente de encruamento $n$ ) e possui características mecânicas ao longo das direções razoavelmente similares (figura 8).
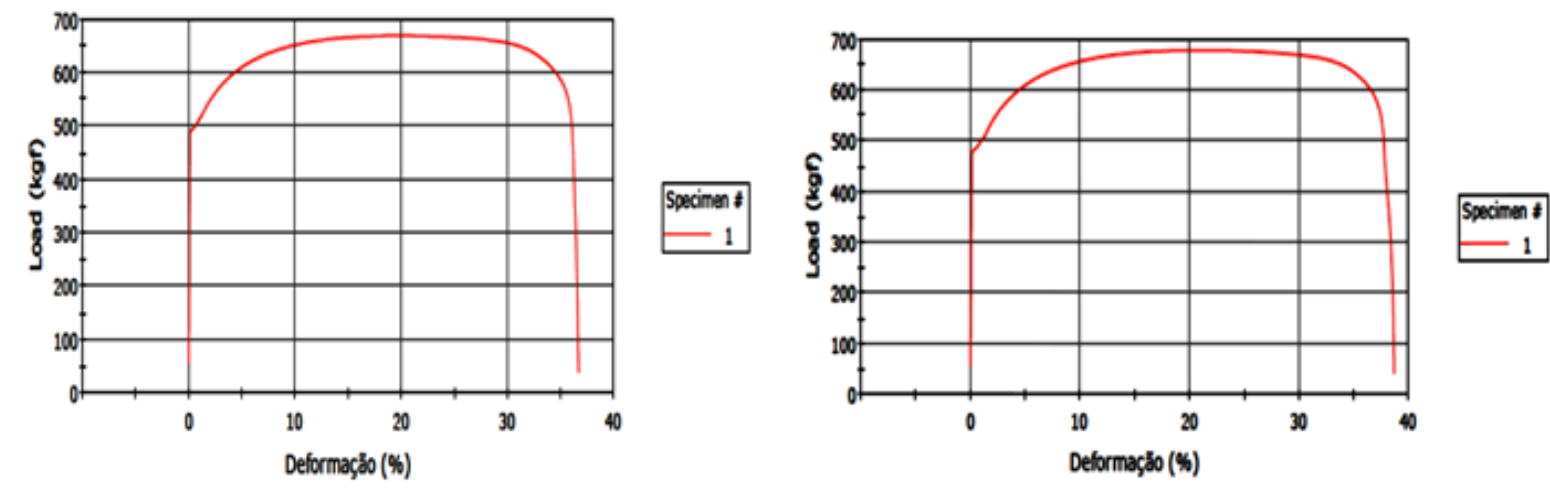

\begin{tabular}{|l|c|c|c|c|c|c|c|}
\hline AMOSTRA & ESP & LARG & DIR & LE & LR & LE/LR & ALONG \\
\hline 10163845 & 0,96 & 19,62 & C & 279 & 318 & 87,7 & 41 \\
\hline
\end{tabular}

\begin{tabular}{|c|c|c|c|c|c|c|c|}
\hline AMOSTRA & ESP & LARG & DIR & LE & LR & LE/LR & ALONG \\
\hline 10163845 & 0,97 & 19,69 & L & 273 & 314 & 86,9 & 42 \\
\hline
\end{tabular}

Figura 7. Curva de tensão deformação de engenharia do aço BH 180 recozido, sentido transversal (C) e longitudinal (L).

\begin{tabular}{|c|c|c|c|c|c|c|c|c|c|c|c|c|c|c|}
\hline \multicolumn{15}{|c|}{ Tração BH } \\
\hline \multirow{2}{*}{ Amostra } & \multicolumn{6}{|c|}{ Primeiro estágio } & \multicolumn{7}{|c|}{ Segundo estágio } & \multirow{2}{*}{$\mathrm{BH}(\mathrm{MPa})$} \\
\hline & \multicolumn{2}{|c|}{ ESP $1(\mathrm{~mm})$} & \multicolumn{3}{|c|}{ LARG $1(\mathrm{~mm})$} & IE 1 (MPa) & \multicolumn{3}{|c|}{ ESP 2 (mm) } & \multicolumn{3}{|c|}{ LARG $2(\mathrm{~mm})$} & IE 2 (MPa) & \\
\hline \multirow[t]{2}{*}{10163845} & \multicolumn{2}{|c|}{0,97} & \multicolumn{3}{|c|}{19,71} & 268 & \multicolumn{3}{|c|}{0,9} & \multicolumn{3}{|c|}{19,26} & 268 & 0 \\
\hline & \multicolumn{14}{|c|}{ Lankford (RN) } \\
\hline \multirow{2}{*}{ Amostra } & \multirow{2}{*}{ ESP } & \multirow{2}{*}{ LARG } & \multicolumn{3}{|c|}{ Transversal $\left(90^{\circ}\right)$} & \multicolumn{3}{|c|}{ Longitudinal $\left(0^{\circ}\right)$} & \multicolumn{4}{|c|}{ Diagonal $\left(45^{\circ}\right)$} & \multirow{2}{*}{ R Normal } & \multirow{2}{*}{ Valor de N } \\
\hline & & & AL UNIF & $\mathbf{R}$ & $N$ & AL UNIF & $\mathbf{R}$ & $\mathbf{N}$ & AL & JNIF & $\mathbf{R}$ & $\mathbf{N}$ & & \\
\hline 10163845 & 1 & 19,7 & 20,13 & 2,1 & 2,6 & 20,05 & 2 & 2,7 & 20 & 05 & 1,7 & 3 & 1,88 & 2,65 \\
\hline \multicolumn{15}{|c|}{ Dureza (HRB) } \\
\hline \multicolumn{3}{|c|}{ Amostra } & \multicolumn{3}{|c|}{ Medição 1} & \multicolumn{4}{|c|}{ Medição 2} & \multicolumn{4}{|c|}{ Medição 3} & Média \\
\hline \multicolumn{3}{|c|}{10163845} & \multicolumn{3}{|c|}{40} & \multicolumn{4}{|c|}{40} & \multicolumn{4}{|c|}{41} & 40,3 \\
\hline
\end{tabular}

Figura 8. Resultados da tração $\mathrm{BH}$ após recozimento, resultados de anisotropia (R) e expoente de encruamento $(n)$ e resultados de dureza na escala Rockwell B. 


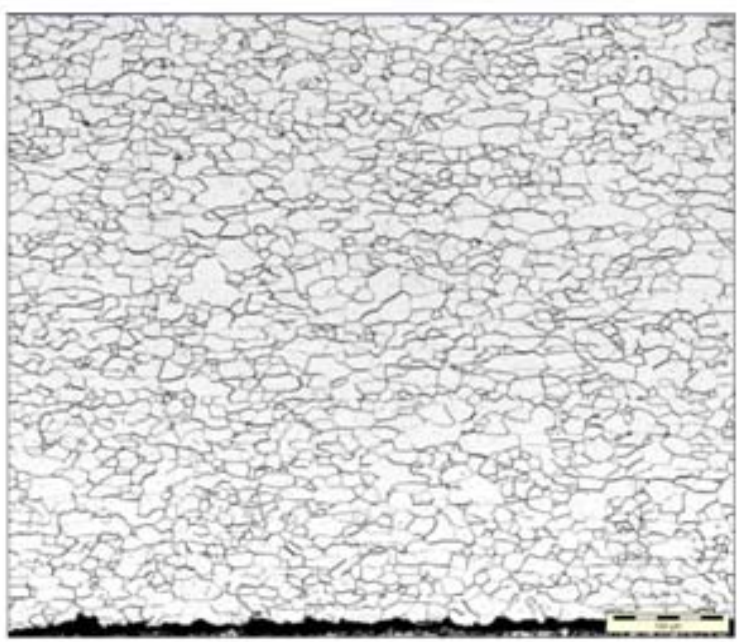

Figura 9. Fotos das microestruturas a partir das amostras recozidas. Ampliação de 1000 e 100 vezes.

Após a laminação de encruamento é percebida uma considerável melhoria nas propriedades de interesse, que para esse aço é o incremento de resistência mecânica após o processo de estampagem e pintura (figura 10). O limite de escoamento, resistência e dureza assumiram valores bem próximos da condição de laminado a quente, porém $\mathrm{o}$ valor $\mathrm{BH}$ apresentou um considerável ganho, evidenciado pelo resultado da amostra 10163136 (figura 11). A queda no valor de $n$ mostra que o material possui uma parcela de encruamento. Fato este que pode ser observado também na análise da microestrutura da amostra, verificando o direcionamento (alongamento) dos grãos no sentido de laminação (figura 12). 0 valor $r$ apresenta condições favoráveis ao processo de estampagem.
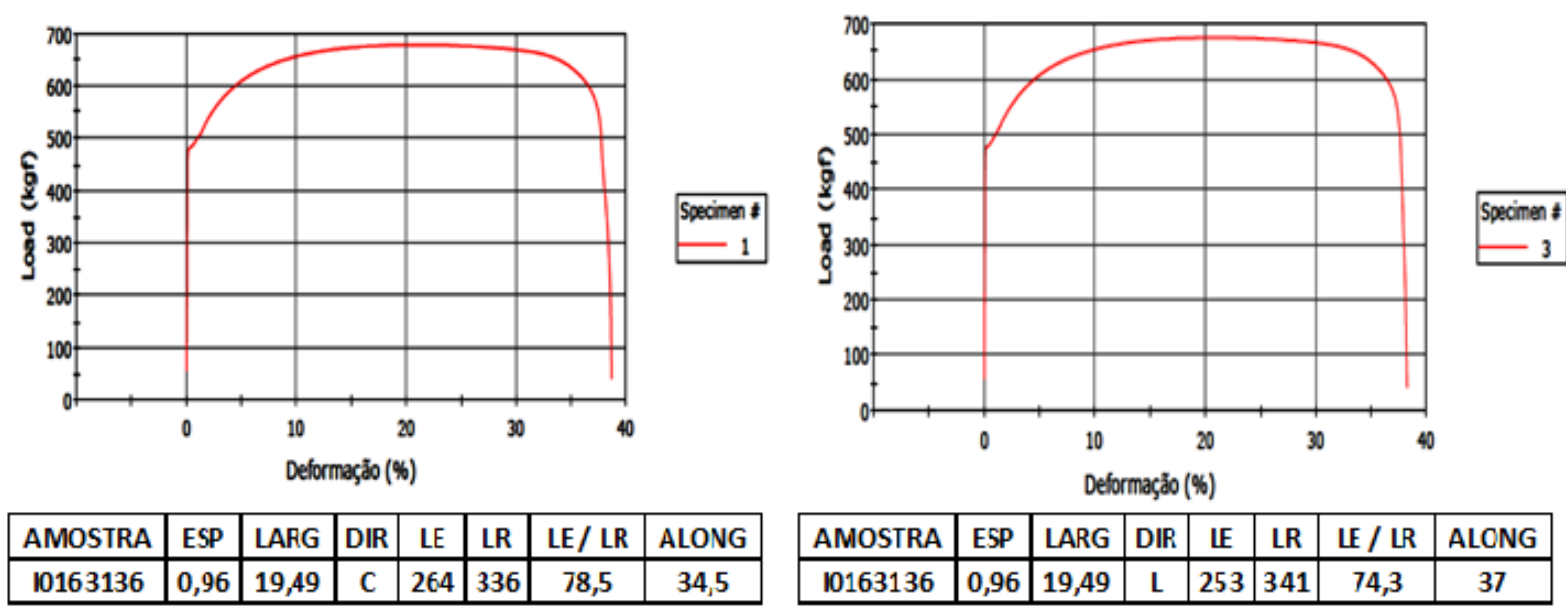

Figura 10. Curva de tensão deformação de engenharia do aço BH 180 encruado, sentido transversal (C) e longitudinal (L). 


\begin{tabular}{|c|c|c|c|c|c|c|c|c|c|c|c|c|c|}
\hline \multicolumn{14}{|c|}{ Tração BH } \\
\hline \multirow{2}{*}{ Amostra } & \multicolumn{6}{|c|}{ Primeiro estágio } & \multicolumn{6}{|c|}{ Segundo estágio } & \multirow{2}{*}{$\mathrm{BH}$ (MPa) } \\
\hline & \multicolumn{2}{|c|}{ ESP 1 (mm) } & \multicolumn{2}{|c|}{ LARG $1(\mathrm{~mm})$} & & LE 1 (MPa) & \multicolumn{2}{|c|}{ ESP $2(\mathrm{~mm})$} & \multicolumn{3}{|c|}{ LARG $2(\mathrm{~mm})$} & LE 2 (MPa) & \\
\hline \multirow[t]{2}{*}{10163136} & \multicolumn{2}{|r|}{0,96} & \multicolumn{2}{|c|}{19,95} & & 255 & \multicolumn{2}{|c|}{0,95} & \multicolumn{3}{|c|}{19,79} & 311 & 56 \\
\hline & \multicolumn{13}{|c|}{ Lankford (RN) } \\
\hline \multirow{2}{*}{ Amostra } & \multirow{2}{*}{ ESP } & \multirow{2}{*}{ LARG } & \multicolumn{3}{|c|}{ Transversal $\left(90^{\circ}\right)$} & \multicolumn{3}{|c|}{ Longitudinal $\left(0^{\circ}\right)$} & \multicolumn{3}{|c|}{ Diagonal $\left(45^{\circ}\right)$} & \multirow{2}{*}{ R Normal } & \multirow{2}{*}{ Valor de $\mathrm{N}$} \\
\hline & & & AL UNIF & $\mathbf{R}$ & $\mathbf{N}$ & AL UNIF & $\mathbf{R}$ & $\mathrm{N}$ & AL UNIF & $\mathbf{R}$ & $\mathbf{N}$ & & \\
\hline 10163136 & 1 & 19,9 & 21,13 & 2 & 1,9 & 21,35 & 1,8 & 1,9 & 21,1 & 1,4 & 2 & 1,72 & 1,84 \\
\hline \multicolumn{14}{|c|}{ Dureza (HRB) } \\
\hline \multicolumn{3}{|c|}{ Amostra } & \multicolumn{3}{|c|}{ Medição 1} & \multicolumn{3}{|c|}{ Medição 2} & \multicolumn{4}{|c|}{ Medição 3} & Média \\
\hline \multicolumn{3}{|c|}{10163136} & \multicolumn{3}{|c|}{58} & \multicolumn{3}{|c|}{59} & \multicolumn{4}{|c|}{59} & 58,7 \\
\hline
\end{tabular}

Figura 11. Resultados da tração $\mathrm{BH}$ após encruamento, resultados de anisotropia (R) e expoente de encruamento $(n)$ e resultados de dureza na escala Rockwell $B$.
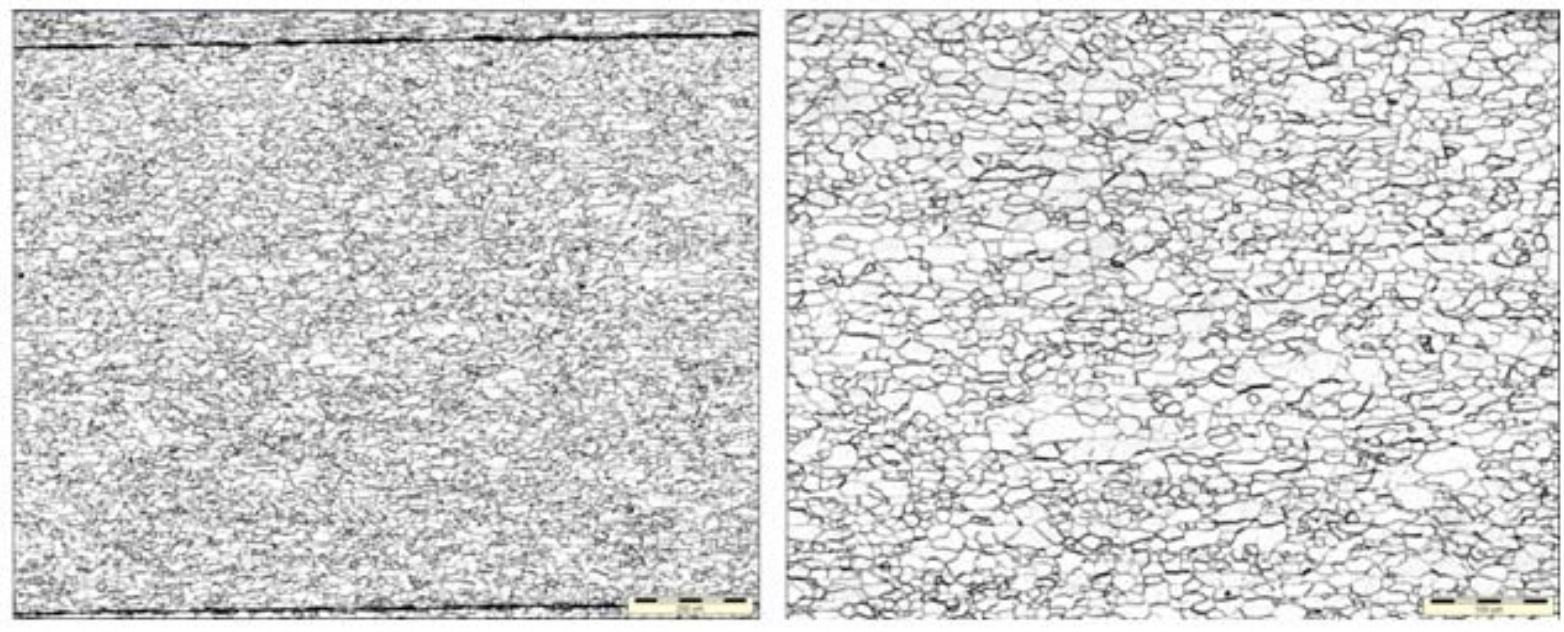

Figura 12. Fotos das microestruturas a partir das amostras após encruamento. Ampliação de 500 e 1000 vezes.

Os valores de alongamento medidos no ensaio sofreram variações consideráveis após processo de laminação a frio e laminação de encruamento, o que já era esperado, pois tal processo reflete diretamente na redução da capacidade de alongamento, uma vez que o material apresenta maior volume de discordâncias, que por sua vez dificultam o escoamento. A pequena diminuição no alongamento com a aplicação de maior percentual de redução no passe de encruamento é condizente com o esperado.

As medições do coeficiente de anisotropia não indicaram diferenças estatísticas entre as combinações experimentais do laminado a quente, sendo a variação observada atribuída ao erro do ensaio de tração. Já para os processos de laminação a frio e recozimento há diferenças significativas causadas pelas características microestruturais adquiridas em cada um desses processos. Na laminação de encruamento há uma adequação (ajuste) dessa propriedade visando patamares favoráveis ao processo de estampagem.

Foram obtidos valores de coeficiente de encruamento (valor $n$ ) para todas as posições amostradas. Sua análise revelou sofrer influência da variação no percentual de encruamento, uma vez que com o avanço no campo plástico, novos mecanismos de deformação passam a atuar, modificando o valor $\mathrm{n}$. Isto garante boa 
capacidade do material ser estirado sem sofrer estricção, ao ter sua seção central ao comprimento deformado encruado rapidamente à medida que se alonga, redistribuindo tensões para regiões vizinhas à seção. Para os tempos de recozimento aplicados ao aço, não há variação significativa no valor $n$, uma vez que todos os materiais sofreram semelhantes níveis de recuperação e recristalização.

\section{CONCLUSÃO}

Obteve-se uma dispersão considerável nos valores de $\mathrm{BH}$, após análise de variância entre as combinações de processo. Os valores de $\mathrm{BH}$ se anularam nos processos de laminação a frio devido a incapacidade de 0 material suportar novas deformações, portanto não poderia ser submetido a um novo processo de conformação mecânica (Estampagem) e no processo de recozimento visto que a morfologia dos grãos e a recuperação elástica impediram o incremento de resistência após deformação e envelhecimento. Os índices de $\mathrm{BH}$ obtidos também não se mostraram elevados demais (40 a $60 \mathrm{MPa}$ ), diminuindo a velocidade de envelhecimento do aço à temperatura ambiente. Este aspecto é importante para a indústria automotiva, onde estoques de matéria-prima ocasionalmente atingem períodos mais longos do que o almejado.

Observa-se que após a laminação de encruamento e consequente eliminação do patamar definido de escoamento, houve uma melhora nas propriedades mecânicas principalmente no valor $\mathrm{BH}, \mathrm{r}$ e $\mathrm{n}$ evidenciando a importância do processo para aços que serão submetidos ao processo de estampagem.

\section{Agradecimentos}

A Unileste pelo fornecimento de material e equipamento.

Ao professor Jetson Lemos Ferreira, pelo auxilio e interpretação de dados coletados durante a pesquisa.

\section{REFERÊNCIAS}

1 ARAUJO, V. H. L; MATTOS, G. A; BAPTISTA, A. L. B; RIBAS, P. R. F. A Metalografia Colorida utilizada em Análise Microestrutural de Materiais para Estampagem Empregados na Indústria Automobilística. In: $56^{\circ}$ Congresso Anual Internacional da ABM, 2001, Belo Horizonte. 56 Congresso Anual Internacional da ABM, 2001.

2 MACHADO, F. A. Influência do grau de deformação e do tratamento "Bake Hardening" na propagação de trinca por fadiga em dois aços bifásicos usados em rodas automobilísticas. 2005. Dissertação (Mestrado em Engenharia de Materiais)Universidade Estadual de Minas Gerais, Belo Horizonte, 2005.

3 CASTELO BRANCO DIAS, G. Estudo do efeito "bake hardening" nos aços BH180 e $\mathrm{BH} 220$ galvanizados pelo processo de imersão a quente. Dissertação (Mestrado) Universidade Estadual Paulista, Faculdade de Engenharia de Guaratinguetá, 2011.

4 GORNI, A. A. Novos aços ferríticos garantem alta resistência e conformabilidade a chapas finas laminadas a frio. Corte \& Conformação de Metais, p. 34-36, set. 2010.

5 ZHANG, J. et al. Bake hardening behavior of TRIP and DP steels. Journal of University of Science and Technology, Beijing, v. 15, n. 2, p. 132, Apr. 2008.

6 MACHADO, F. A. Influência do grau de deformação e do tratamento "Bake Hardening" na propagação de trinca por fadiga em dois aços bifásicos usados em rodas automobilísticas. 2005. Dissertação (Mestrado em Engenharia de Materiais)Universidade Estadual de Minas Gerais, Belo Horizonte, 2005. 